\title{
Stock market return distributions: from past to present
}

\author{
S. Drożdż ${ }^{1,2}$, M. Forczek ${ }^{1}$, J. Kwapień ${ }^{1}$, P. Oświęcimka ${ }^{1}$, R. Rak ${ }^{2}$ \\ ${ }^{1}$ Institute of Nuclear Physics, Polish Academy of Sciences, PL-31-342 Kraków, \\ Poland \\ 2 Institute of Physics, University of Rzeszów, PL-35-959 Rzeszów, Poland
}

\begin{abstract}
We show that recent stock market fluctuations are characterized by the cumulative distributions whose tails on short, minute time scales exhibit power scaling with the scaling index $\alpha>3$ and this index tends to increase quickly with decreasing sampling frequency. Our study is based on high-frequency recordings of the S\&P500, DAX and WIG20 indices over the interval May 2004 - May 2006. Our findings suggest that dynamics of the contemporary market may differ from the one observed in the past. This effect indicates a constantly increasing efficiency of world markets.
\end{abstract}

Key words: Financial markets, Inverse cubic power law, $q$-Gaussian distributions, Multifractality

PACS: 89.20.-a, 89.65.Gh, 89.75.-k

The so-called financial stylized facts are among the central issues of econophysics research. Much effort has been devoted on both the empirical and the theoretical level to such phenomena like fat-tailed distributions of financial fluctuations, persistent correlations in volatility, multifractal properties of returns etc. Specifically, the interest in the return distributions can be traced back to an early work of Mandelbrot [1] in which he proposed a Lévy process as the one governing the logarithmic price fluctuations. Much later this issue was revisited in [2] based on data with much better statistics and a new model of exponentially-truncated Lévy flights was introduced. Then, in an extensive systematic study of the largest American stock markets [3] the distribution tails for both the prices and the indices were shown to be power-law with the scaling exponent $\alpha \simeq 3$. The most striking outcome of that study was that despite the fact that the tails were well outside the Lévy-stable regime $(\alpha \leq 2)$, they were apparently stable under time aggregation up to several days for indices and up to a month for stocks. The existence of return distributions with 
scaling tails was also reported in other markets like e.g. London [4], Frankfurt [5], Paris [6], Oslo [7], Tokyo [3], and Hong Kong [3,8] but sometimes with a slightly different value of the scaling index. This empirical property of price and index returns led to the formulation of the so-called inverse cubic power law [3], which was soon followed by an attempt of formulating its theoretical foundation [6] (see also [4]).

Subsequent related study [9] revealed that, opposite to the earlier outcomes of [3, the tail shape of the return distributions might no longer be so stable along time axis. After comparison of the results obtained from the American stock market data in years 1994-95 and in 1998-99, it turned out that in more recent data the scaling tails with $\alpha \simeq 3$ for individual companies are preserved up to the time scales (sampling intervals) $\Delta t$ of less than one hour instead of one month. This earlier crossover for 1998-99 data can easily be seen in Fig. 1. This result was obtained by extending our previous analysis [9] over a set of 1000 largest American companies [10] in order to enable more direct comparison with outcomes of ref. [3] based on the same number of stocks. The inverse cubic scaling is still evident in Fig. 1 for short time scales up to $\Delta t=4 \mathrm{~min}$, but the scaling index starts rising already for data with $\Delta t=16$ min and for longer time scales the tail behaviour is clearly governed by the Central Limit Theorem. The difference of the results for the same market but for different time intervals might suggest that the scaling behaviour is not stable and depends on some crucial factors as, for example, the speed of information processing which constantly increases from past to present.

This possibility can further be examined by considering even more recent data from the American market. First, we look at the S\&P500 index which already was the subject of an analysis in [3]. Our data is a time series of 1 min returns covering the period May 2004 - May 2006 (in [3] the period was 1984-1996). The c.d.f. for this data is presented in Fig. 2(a) for several time scales up to $120 \mathrm{~min}$. The most interesting feature is the lack of inverse cubic scaling even for the shortest one-minute returns: in this case the actual scaling index is slightly above 4 and it systematically increases with decreasing sampling frequency (see Table 1). We leave open here the question what factor underlies the evident absence of the $\alpha \simeq 3$ type of scaling: the dynamics of S\&P500 returns could have changed sufficiently significantly since earlier half of 1990s and the inverse cubic scaling no longer exists or it still exists but is restricted to time scales shorter than 1 minute. That our observation is more universal and can be made for other markets as well one may infer from Fig. 2(b) and Fig. 2(c) presenting c.d.f. for the German index DAX and for the Polish index WIG20, respectively, for the same period of time. While the returns of DAX did not exactly comply with the inverse cubic scaling also in the period 1998-99 9], the ones of WIG20 indeed used to display this kind of behaviour in the past as documented in [11. However, nowadays WIG20 also develops much thinner tails with $\alpha>4$ for $\Delta t=1 \min$ (Table 1). 


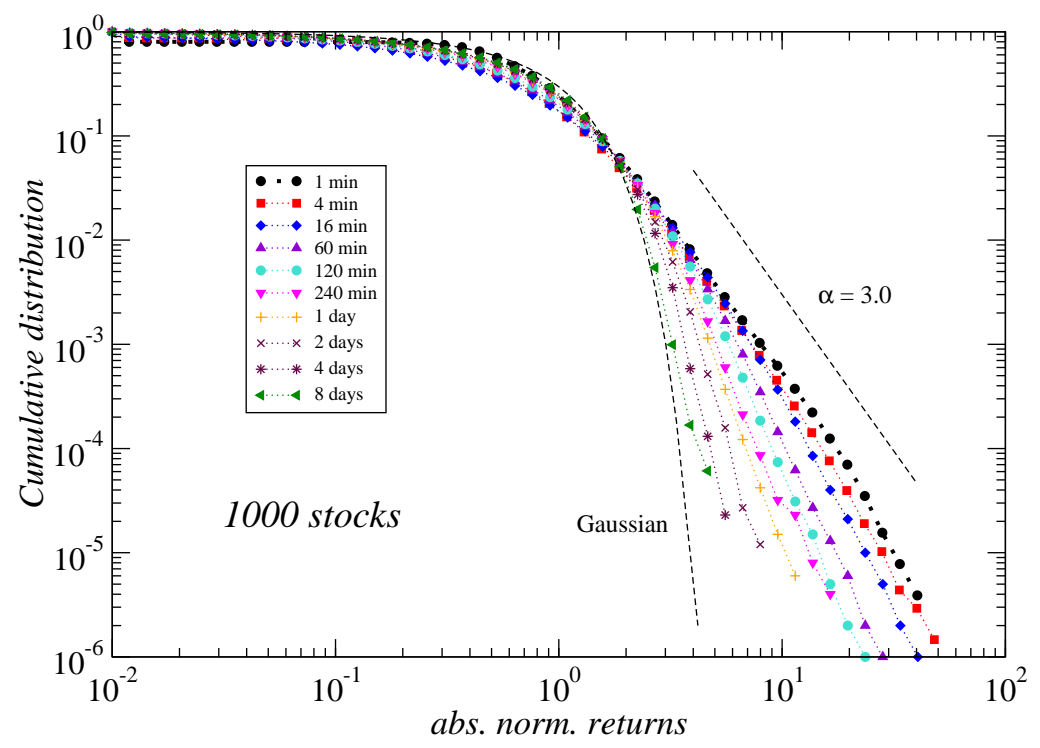

Fig. 1. Cumulative distributions of normalized stock returns averaged over 1000 highly-capitalized American companies in time interval Dec 1997 - Dec 1999 for several different time scales from 1 min to 8 days. Gaussian distribution and inverse cubic scaling are also shown for comparison. Best-fit power index $\alpha$ calculated by means of log-log regression assumes the following values: $3.08 \pm 0.05(\Delta t=1 \mathrm{~min})$, $3.34 \pm 0.05$ (4 min), $4.00 \pm 0.04$ (16 min), $4.60 \pm 0.05$ (60 $\mathrm{min}), 4.95 \pm 0.06(120$ $\min$ ), $4.81 \pm 0.15$ (240 $\min$ ), $5.90 \pm 0.08$ (1 day), $7.18 \pm 0.28$ (2 days), $9.17 \pm 0.22$ (4 days), and $8.32 \pm 0.40$ ( 8 days).

\begin{tabular}{|c||c|c|c|c|c|}
\hline$\Delta t$ & $1 \mathrm{~min}$ & $4 \mathrm{~min}$ & $16 \mathrm{~min}$ & $32 \mathrm{~min}$ & $60 \mathrm{~min}$ \\
\hline \hline S\&P500 & $4.12 \pm 0.12$ & $4.21 \pm 0.08$ & $5.18 \pm 0.21$ & $5.53 \pm 0.20$ & $6.10 \pm 0.35$ \\
\hline DAX & $3.56 \pm 0.12$ & $3.76 \pm 0.05$ & $4.44 \pm 0.16$ & $5.14 \pm 0.26$ & $5.16 \pm 0.66$ \\
\hline WIG20 & $4.28 \pm 0.16$ & $5.24 \pm 0.27$ & $5.81 \pm 0.78$ & $5.61 \pm 0.45$ & $6.30 \pm 0.72$ \\
\hline \hline
\end{tabular}

Table 1

Values of the scaling index $\alpha$ obtained with a log-log regression fit for three different market indices (S\&P500, DAX, and WIG20) from the interval May 2004 - May 2006.

Recent studies [11, showed that in a wide range of returns the financial return distributions can be approximated by a family of $q$-Gaussians [13] with the parameter $q$ depending on the sampling interval $\Delta t$. The $q$-Gaussian distributions follow naturally from the nonextensive statistical mechanics [13,12] and their c.d.f. can be written as [11]

$$
P(X>x)=\mathcal{N}_{q}\left(\frac{\sqrt{\pi} \Gamma\left(\frac{1}{2}(3-q) \beta\right)}{2 \Gamma(\beta) \sqrt{\frac{\mathcal{B}_{q}}{\beta)}}} \pm\left(x-\overline{\mu_{q}}\right)_{2} F_{1}(\alpha, \beta ; \gamma ; \delta)\right)
$$



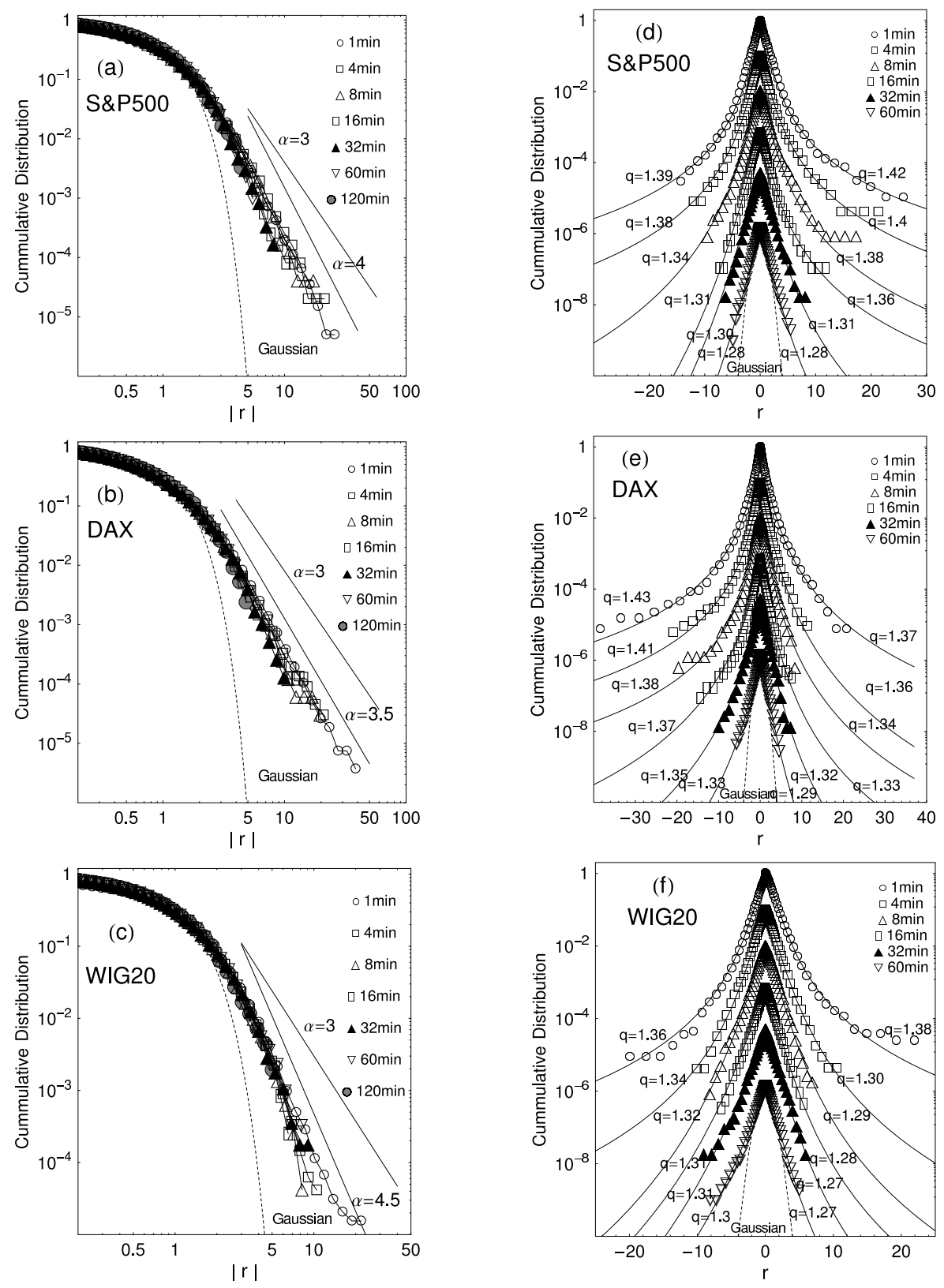

Fig. 2. Cumulative distributions of index returns for American S\&P500 (top row), German DAX (middle row) and Polish WIG20 (bottom row) recorded over the interval May 2004 - May 2006. Data points for sampling intervals from 1 min to 120 min are denoted by different symbols. (Left column) Distributions for normalized returns are shown together with Gaussian distribution and lines corresponding to inverse cubic scaling and, approximately, the actual scaling. (Right column) Experimental distributions are best-fitted by $q$-Gaussians with a free parameter $q$ (fitted values displayed) separately for negative and positive returns. Note the small asymmetry between left and right tails. 


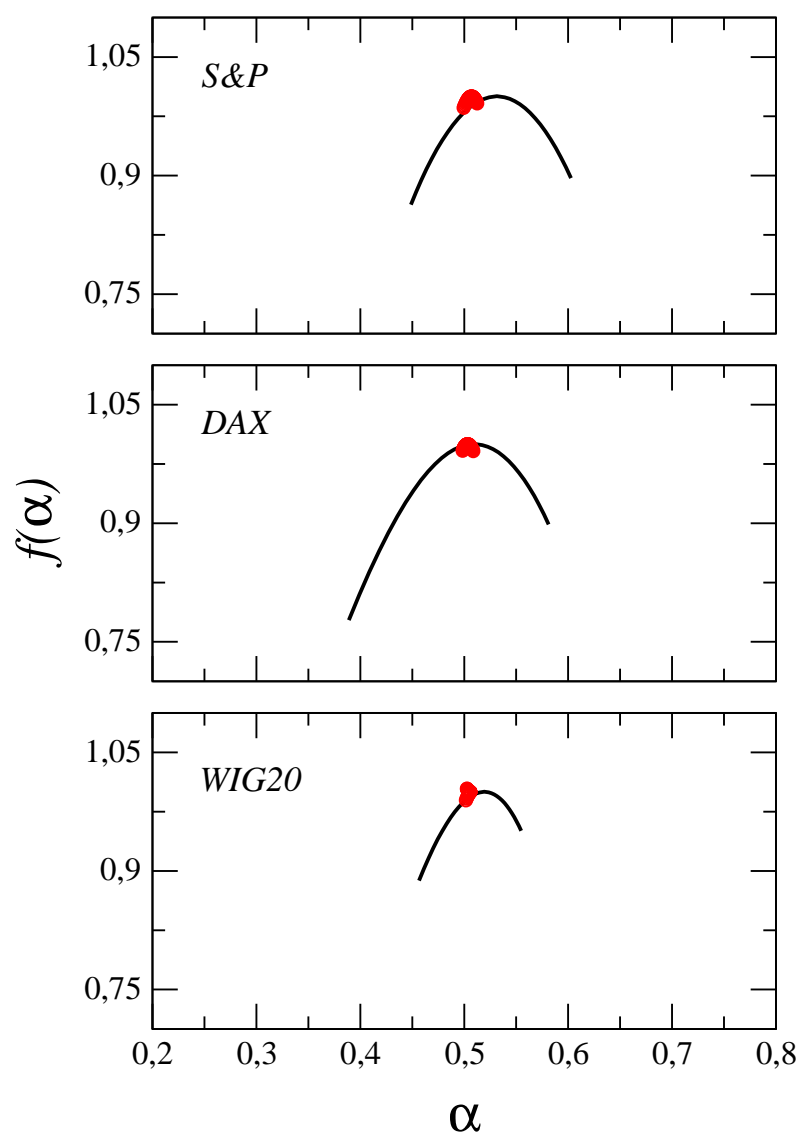

Fig. 3. Singularity spectra for 1 min index returns: S\&P500 (top), DAX (middle), and WIG20 (bottom). In each case, the actual data (solid line) is accompanied by its randomized version averaged over 10 independent realizations (full symbols).

where $\alpha=\frac{1}{2}, \beta=1 /(q-1), \gamma=\frac{3}{2}, \delta=-\mathcal{B}_{q}(q-1)\left(\bar{\mu}_{q}-x\right)^{2}{ }_{2} F_{1}(\alpha, \beta ; \gamma ; \delta)=$ $\sum_{k=0}^{\infty} \frac{\delta^{k}(\alpha)_{k}(\beta)_{k}}{k !(\gamma)_{k}}$ is the Gauss hypergeometric function and $\mathcal{N}_{q}, \bar{\mu}_{q}$ are, respectively, the normalization factor and $q$-mean of the $q$-Gaussian p.d.f. [13]. Fig. 2(d)-2(f) exhibit cumulative distributions of returns for the same three indices with the corresponding best fits in terms of Eq.(1). The theoretical curves are in satisfactory agreement with the data for all the considered values of $\Delta t$. It is noteworthy that, consistently with the left-hand side panels of Fig. 2, the largest values of $q$ are well below $q=3 / 2$, which corresponds to the inverse cubic scaling, and decrease with decreasing sampling frequency towards the classic Gaussian distribution with $q=1$.

Finally, we look at the singularity spectra $f(\alpha)$ (for numerical details of the method see e.g. [15]) of our time series under study. Two cases are considered: original data comprising the full variety of nonlinear temporal correlations (solid lines in Fig. 3) and the randomized data in which all the correlations are removed by shuffling the data points (full symbols in Fig. 3). Since both the nonlinear dependencies and the fat-tailed distributions can be potential sources of multifractality, the data shuffling can give some information of how 
rich is the multiscaling behaviour due to each of these sources [14]15]. In the present context the most interesting feature is that the Gaussian distribution of uncorrelated data is associated with a monofractal $f(\alpha)$ spectrum. Fig. 3 shows the singularity spectra for S\&P500, DAX and WIG20 (top to bottom) together with their randomized-data counterparts. In all three cases, the original data clearly represent multifractal processes with DAX and S\&P500 showing richer multifractality than WIG20. This picture changes completely if we look at the randomized data: we cannot detect sufficiently significant trace of multifractality $(f(\alpha)$ is almost point-like). This is in agreement with the observation that the distribution tails for the contemporary data tend to be thinner than before. This result gives also an additional argument in favour of the statement that the principal (here even the unique) source of multifractal properties of the stock market data are the nonlinear correlations.

In this paper we have shown that c.d.f. for the most recent stock market data represented by the index returns develops tails whose scaling index rises above the value of 3 even for short, minute sampling intervals. This means that contemporary market dynamics significantly differs from the one observed 20 or even 10 years ago and described by the inverse cubic power law [3]. That these changes are a continuous process rather that a sudden transition we infer from the existence of intermediate stages in which the inverse cubic scaling was observed up to medium time scales of tens of minutes but was absent in daily data [9]11. This effect suggests a scenario of constantly increasing market efficiency due to an acceleration of information processing in the world markets [9,16]. The related compression of the range of potential time correlations between consecutive returns in the present analysis finds evidence in a faster convergence towards a Gaussian distribution for aggregated returns. Such a faster convergence indicates weeker time-correlations between returns. Further argument in favour of an increasing stock market efficiency comes from the autocorrelation analysis. The autocorrelation functions calculated exlicitely from the 1 min returns for the three indices considered above drop down to the noise level already for time-lags as small as 1-2 min. This is to be compared to $\sim 5$ min in the period 1998-99 [9], and to $\sim 20$ min which according to ref. [3] was characteristic for the period 1994-95.

\section{References}

[1] B. Mandelbrot, J. Business 36, 294 (1963)

[2] R.N. Mantegna, H.E. Stanley, Nature 376 (1995) 46

[3] P. Gopikrishnan, V. Plerou, L.A. Nunes Amaral, M. Meyer, H.E. Stanley, Phys. Rev. E 60 (1999) 5305; V. Plerou, P. Gopikrishnan, L.A.N. Amaral, M. Meyer, H.E. Stanley, Phys. Rev. E 60 (1999) 6519 
[4] J.D. Farmer, F. Lillo, Quant. Finance 4 (2004) C7; J.D. Farmer, L. Gillemot, F. Lillo, S. Mike, A. Sen, Quant. Finance 4 (2004) 383

[5] T. Lux, Appl. Financial Economics 6 (1996) 463

[6] X. Gabaix, P. Gopikrishnan, V. Plerou, H.E. Stanley, Nature 423 (2003) 267

[7] J.A. Skjeltorp, Physica A 283 (2000) 486

[8] Z.F. Huang, Physica A 287 (2000) 405

[9] S. Drożdż, J. Kwapień, F. Grümmer, F. Ruf, J. Speth, Acta Phys. Pol. B 34 (2003) 4293, cond-mat/0208240

[10] See http://www.taq.com

[11] R. Rak, S. Drożdż, J. Kwapień, Physica A 374 (2007) 315

[12] C. Tsallis, C. Anteneodo, L. Borland, R. Osorio, Physica A 324 (2003) 89

[13] C. Tsallis, R.S. Mendes, A.R. Plastino, Physica A 261 (1998) 534

[14] K. Matia, Y. Ashkenazy, H.E. Stanley, Europhys. Lett. 61 (2003) 422

[15] J. Kwapień, P. Oświęcimka, S. Drożdż, Physica A 350 (2005) 466

[16] J. Kwapień, S. Drożdż, J. Speth, Physica A 337 (2004) 231 